\title{
A reanalysis of "Two types of asynchronous activity in networks of excitatory and inhibitory spiking neurons"
}

\section{[version 1; peer review: 2 approved]}

\author{
Rainer Engelken ${ }^{1,2^{*}}$, Farzad Farkhooi ${ }^{* *}$, David Hansel ${ }^{4}$, Carl van Vreeswijk ${ }^{4}$, \\ Fred Wolf 1,2
}

\begin{abstract}
${ }^{1}$ Max Planck Institute for Dynamics and Self-Organization (MPI-DS), Bernstein Center for Computational Neuroscience Göttingen, Faculty of Physics,, University of Göttingen, Göttingen, Germany

${ }^{2}$ Collaborative Research Center 889, University of Göttingen, Göttingen, 37099, Germany

${ }^{3}$ Institut für Mathematik, Technische Universität Berlin and Bernstein Center for Computational Neuroscience, Berlin, Germany ${ }^{4}$ Cerebral Dynamics, Learning and Memory Research Group, Center for Neurophysics, Physiology and Pathology, CNRS UMR8119, Université Paris Descartes, Paris, France

* Equal contributors
\end{abstract}

\section{V1 First published: 22 Aug 2016, 5:2043 \\ https://doi.org/10.12688/f1000research.9144.1}

Latest published: 22 Aug 2016, 5:2043

https://doi.org/10.12688/f1000research.9144.1

\section{Abstract}

Neuronal activity in the central nervous system varies strongly in time and across neuronal populations. It is a longstanding proposal that such fluctuations generically arise from chaotic network dynamics. Various theoretical studies predict that the rich dynamics of rate models operating in the chaotic regime can subserve circuit computation and learning. Neurons in the brain, however, communicate via spikes and it is a theoretical challenge to obtain similar rate fluctuations in networks of spiking neuron models.

A recent study investigated spiking balanced networks of leaky integrate and fire (LIF) neurons and compared their dynamics to a matched rate network with identical topology, where single unit inputoutput functions were chosen from isolated LIF neurons receiving Gaussian white noise input. A mathematical analogy between the chaotic instability in networks of rate units and the spiking network dynamics was proposed.

Here we revisit the behavior of the spiking LIF networks and these matched rate networks. We find expected hallmarks of a chaotic instability in the rate network: For supercritical coupling strength near the transition point, the autocorrelation time diverges. For subcritical coupling strengths, we observe critical slowing down in response to small external perturbations. In the spiking network, we found in contrast that the timescale of the autocorrelations is insensitive to the coupling strength and that rate deviations resulting from small input

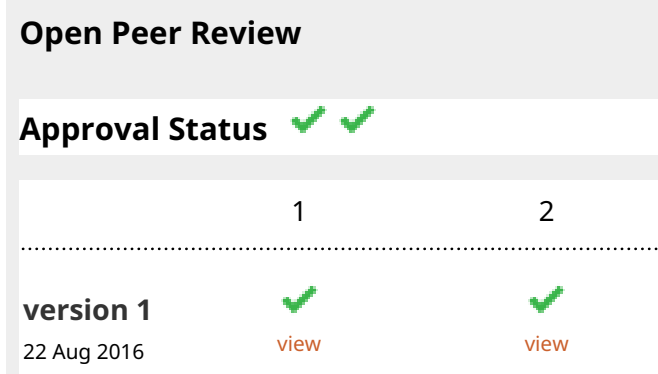

1. Jonathan Touboul, CIRB - Collège de France and Inria Mycenae, Paris, France

2. Maoz Shamir, Ben-Gurion University of the Negev., Be'er-Sheva, Israel

Any reports and responses or comments on the article can be found at the end of the article. 
perturbations rapidly decay. The decay speed even accelerates for increasing coupling strength.

In conclusion, our reanalysis demonstrates fundamental differences between the behavior of pulse-coupled spiking LIF networks and rate networks with matched topology and input-output function. In particular there is no indication of a corresponding chaotic instability in the spiking network.

Keywords

rate chaos, balanced state, mean field theory, network dynamics

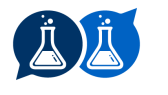

This article is included in the Preclinical

Reproducibility and Robustness gateway.

This article is included in the Max Planck Society

collection.

Corresponding author: David Hansel (david.hansel@parisdescartes.fr)

Competing interests: No competing interests were disclosed.

Grant information: The work of DH and CVV was partially supported by grants ANR-13-BSV4-0014-03 - BALAV1 and ANR-14-NEUC-000101-BASCO and performed in the framework of the France-Israel Laboratory of Neuroscience (FILN). FF was supported by the BMBF, FKZ 01GQ 1001B. RE and FW received funding from Evangelisches Studienwerk Villigst, DFG through CRC 889 and Volkswagen Foundation.

Copyright: $\odot 2016$ Engelken $\mathrm{R}$ et al. This is an open access article distributed under the terms of the Creative Commons Attribution License, which permits unrestricted use, distribution, and reproduction in any medium, provided the original work is properly cited.

How to cite this article: Engelken R, Farkhooi F, Hansel D et al. A reanalysis of "Two types of asynchronous activity in networks of excitatory and inhibitory spiking neurons" [version 1; peer review: 2 approved] F1000Research 2016, 5:2043

https://doi.org/10.12688/f1000research.9144.1

First published: 22 Aug 2016, 5:2043 https://doi.org/10.12688/f1000research.9144.1 


\section{Introduction}

Slow neural dynamics are believed to be important for behavior, learning and memory (Churchland \& Shenoy, 2007; Fee \& Goldberg, 2011; Murray et al., 2014). Rate models operating in the chaotic regime show rich dynamics at the scale of hundreds of milliseconds and provide remarkable learning capabilities (Barak et al., 2013; Sussillo \& Abbott, 2009; Toyoizumi \& Abbott, 2011). Understanding the conditions of such a transition to chaos in more detailed network models has recently attracted a lot of interest (Harish \& Hansel, 2015; Kadmon \& Sompolinsky, 2015). However, neurons in the brain communicate via spikes and it is a challenge in computational neuroscience to obtain similar slow rate dynamics in networks of spiking neuron models.

This question was recently addressed in a paper by Ostojic (2014) published in Nature Neuroscience (Ostojic, 2014). It argues that an "unstructured, sparsely connected network of model spiking neurons can display two fundamentally different types of asynchronous activity". When the synaptic strength is increased, networks of leaky integrate-and-fire (LIF) neurons would undergo a transition from the "well-studied asynchronous state, in which individual neurons fire irregularly at constant rates" to another "heterogeneous asynchronous state" in which "the firing rates of individual neurons fluctuate strongly in time and across neurons" (Ostojic, 2014). These two regimes would differ in an essential manner, the rate dynamics being chaotic beyond the phase transition. Finding a transition to chaotic slow-varying rate dynamics in spiking networks in such a simple model would be an important step towards an understanding of the computations underlying behavior and learning and would fill a gap in the current understanding of network dynamics. Here we re-examine the behavior of random LIF networks and demonstrate that there is no such phase transition to chaos in the spiking network analyzed in (Ostojic, 2014). While we confirm the observed deviation from the mean field theory description that assumes uncorrelated Gaussian fluctuations in time and among neurons, we controvert the validity of the presented analysis. We provide a series of tests of dynamical behavior that refute the existence of a chaotic instability and show that the analogy between the spiking network and the rate network is conceptually misleading and mathematically flawed.

The paper (Ostojic, 2014) starts with simulations of a network of LIF neurons for different values of the synaptic strength, J, while all other parameters are fixed to specific values. It is observed that the population mean firing rate of the neurons, $v_{0}$, is well described by a mean field calculation only below a certain coupling strength $\mathrm{J}^{*}$. At this value, the average firing rate starts to deviate from the mean field prediction more than 5\%. (Figure 1a in (Ostojic, 2014), denoted Figure P1a; hereafter figures in (Ostojic, 2014) are denoted by their numbers preceded by a "P"). In (Ostojic, 2014), it was claimed that the "classical" asynchronous state exhibits an instability at $\mathrm{J}=\mathrm{J}^{*}$. Above $\mathrm{J}^{*}$ the dynamics would still be asynchronous, but in a way which would be essentially different from the "classical" asynchronous state. To assess this claim, the author replaced the full dynamics of the spiking LIF network by a rate model of similar connectivity, the "Poisson network". Simulations indicate that as J increases, there is a value, $\mathrm{J}=\mathrm{J}$, at which the dynamics of the latter undergo a phase transition between a state in which the rates are constant in time (fixed point) and a state in which they fluctuate chaotically with long network generated time-scales. The author then derives an equation for a critical value $J_{c}$ which is in agreement with the simulations of the Poisson model. For the parameters used in Figure P1 and P2 the value of $\mathrm{J}_{\mathrm{c}}$ is rather close to $\mathrm{J}^{*}$. Apparently the author felt that this similarity, gives sufficient reason to justify two conclusions: (i) in the LIF network an instability occurs near $\mathrm{J}^{*}$ which is of the same nature as the one occurring at $\mathrm{J}_{\mathrm{c}}$ in the Poisson network. (ii) The asynchronous states below and above $\mathrm{J}^{*}$ are essentially different in the LIF network.

However, as we now show, the reported agreement between the predicted transition at $\mathrm{J}_{\mathrm{c}}$ and the spiking network simulation results is coincidental and only valid for the chosen parameters used in the paper (Ostojic, 2014) but not in general. We start by providing two counter-examples to statements (i) and (ii).

\section{Methods and results}

Our first counter-example is the LIF model considered in (Ostojic, 2014), we take $\mathrm{N}=40000$ neurons and $\mathrm{C}=4000$ synapses per neuron instead of $\mathrm{N}=10000$ and $\mathrm{C}=1000$ (all other parameters as in Figure P1, except for the network size, keeping the connection probability constant). The population firing rate, $v_{0}(\mathrm{~J})$, is plotted in Figure 1a. It deviates from the mean field prediction at $\mathrm{J}^{*} \cong 0.3 \mathrm{mV}$ by more than $5 \%$. Nonetheless, the critical point in the corresponding Poisson rate network is $\mathrm{J}_{\mathrm{c}} \cong 0.96 \mathrm{mV}$ and thus it is more than three times larger than $\mathrm{J}^{*}$.

Our second counter-example is the LIF network of Figure P1 and P2 with the same parameters except for the delay, $\Delta$. We note that the delay does not affect the existence of the asynchronous state and importantly plays no role in the mathematical considerations of Ostojic (2014). As these yield identical results irrespective of delay we consider the simplest case: $\Delta=0 \mathrm{~ms}$. Strikingly, the spiking network shows no longer a large deviation from the meanfield prediction (Figure 1a). However, the proposed analogy with the Poisson rate network still predicts that a deviation should occur at $\mathrm{J}^{*} \cong 0.49 \mathrm{mV}$, since the transition to chaos in the Poisson network is independent of the delay. The author seems to be somewhat aware of this discrepancy. Indeed, it is stated in the Online Methods that delays must be larger than the refractory period, because "if the delays are shorter, spikes that reach a neuron while it is refractory do not have an effect and the overall coupling is effectively reduced" (Ostojic, 2014). If this was correct, this effective reduction should be reflected in the formula for predicting $\mathrm{J}_{\mathrm{c}}$ (Equation 16). This is not the case: the latter does not depend on $\Delta$. In addition, the spiking network for $\Delta=0 \mathrm{~ms}$ in fact exhibits no increased level of network synchrony measured by the common synchrony measure $\chi$ (Figure 2a) (Hansel \& Mato, 2003).

It is also argued in the paper (Ostojic, 2014) that the results plotted in Figure P3a and b support the analogy between the rate dynamics of the Poisson model and the dynamics of the LIF network. However, the comparison made in this figure is conceptually misleading. In the Poisson model, the rate as a function of time is an unequivocally defined quantity. It is the dynamical variable of the model and the time scale over which the rate fluctuates for strong enough coupling is fully determined by these dynamics. 

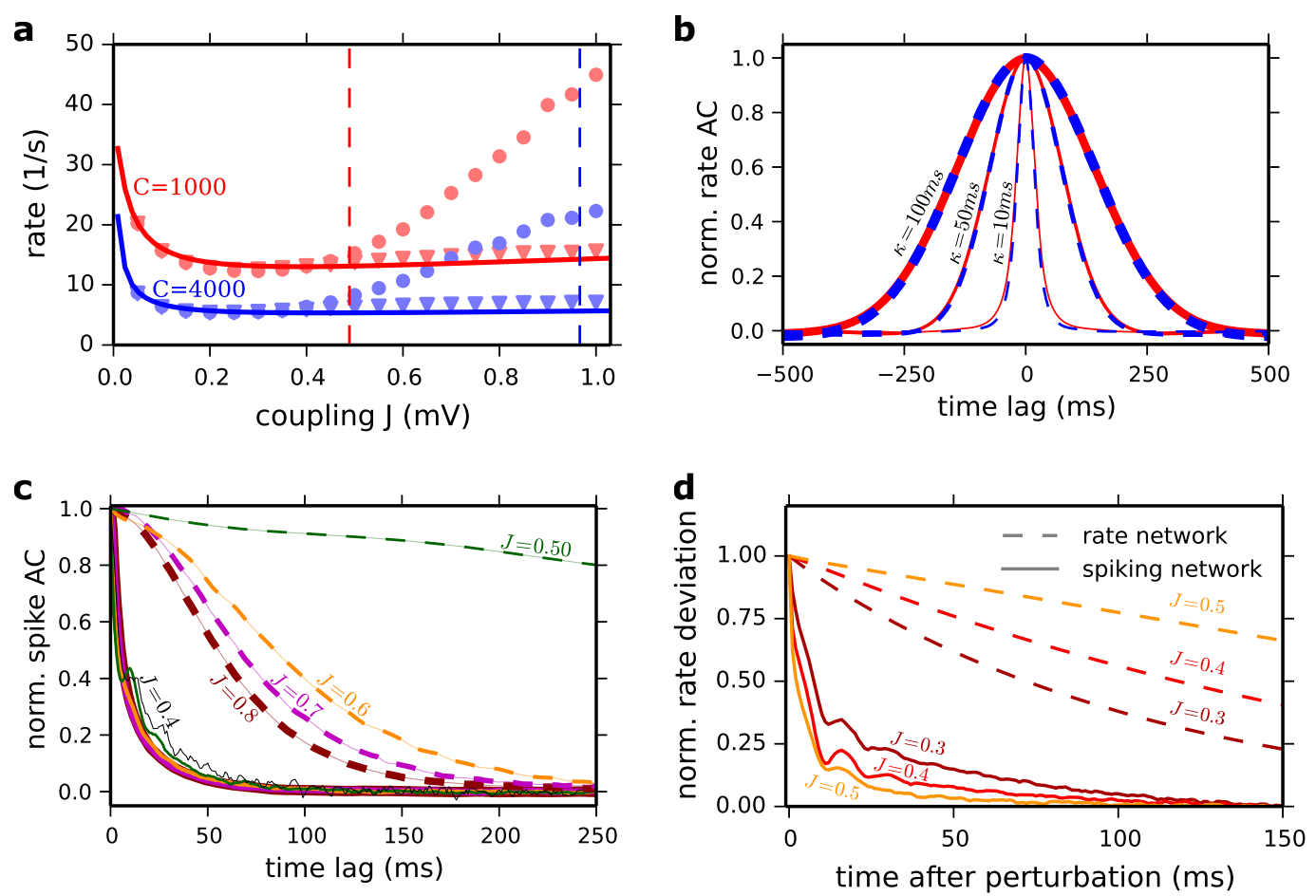

Figure 1. (a) Population averaged firing rate in the network vs. coupling strength J. Solid lines: Ricciardi mean field for $\mathrm{C}=1000$ (red) and $\mathrm{C}=4000$ (blue). Predictions for $\mathrm{J}_{\mathrm{c}}$ (Equation 16) are indicated by the corresponding dashed vertical lines. Simulation results (event-based simulation implemented in Julia programing language) are also plotted. Dots: $\Delta=0.55 \mathrm{~ms}$ synaptic delay. Triangles: $\Delta=0.0 \mathrm{~ms}$. Results for $\mathrm{C}=1000, \mathrm{~N}=10000$ (red marker) and $\mathrm{C}=4000$ and $\mathrm{N}=40000$ (blue marker). (b) Averaged normalized AC of neuronal rate functions for $\mathrm{J}=$ $0.8 \mathrm{mV}$ and $\mathrm{C}=1000$ (red) and $\mathrm{C}=4000$ (dashed blue) LIF networks. The rate functions were computed by filtering the spike trains of the neurons ( $1 \mathrm{~ms}$ time bin) with a Gaussian filter with $10 \mathrm{~ms}$ (the thinnest lines), $50 \mathrm{~ms}$ (moderated lines) and $100 \mathrm{~ms}$ (the thickest lines) standard deviation. (c) Autocorrelation function of the spike trains (no filtering) normalized to the second pick. Solid lines: LIF network. Dashed lines: Poisson network. The results are shown for $\mathrm{J}=0.5 \mathrm{mV}$ (dark green), $\mathrm{J}=0.6 \mathrm{mV}$ (dark orange), $\mathrm{J}=0.7 \mathrm{mV}$ (magenta) and $\mathrm{J}=0.8 \mathrm{mV}$ (dark red). For the LIF the AC is also shown for $\mathrm{J}=0.4 \mathrm{mV}$ (solid black). To compute the ACs for the Poisson network we simulated a network for $100 \mathrm{~s}$ (time step $1 \mathrm{~ms}$ ) and averaged the results over 40 realizations of the initial conditions. The network size is $\mathrm{N}=100000$ for $0.5 \leq \mathrm{J} \leq 0.6 \mathrm{mV}$ and $\mathrm{N}=10000$ for $\mathrm{J}>0.6 \mathrm{mV}$. For the LIF network we averaged spike autocorrelation of 3000 randomly chosen neurons with a $1 \mathrm{~ms}$ bin following Equation 23 in the paper. All parameters are as in Figure P3. (d) Subcritical behavior of the systems. Rate network and spiking network are both perturbed in the constant feed-forward input current $\mu_{0}$ in the least stable direction of the linearized rate dynamics (Equation 16) for different coupling strengths $\mathrm{J}$. The resulting rate deviation is projected onto the perturbation direction. Dashed lines reflect the normalized decay of this perturbation in the rate network and the solid lines those of the spiking network (averaged over 1.42 million perturbations). The perturbation was applied to the constant feed-forward input $\mu_{0}$ for $2 \mathrm{~ms}$ where the standard deviation of the perturbation vector was $1 \mathrm{mV}$. Longer perturbation durations ( $10 \mathrm{~ms}$ ) and weaker perturbation strengths (standard deviation $0.1 \mathrm{mV}$ ) gave very similar results (not shown). Perturbation direction, strength, duration and network realization were exactly the same for rate and spiking network. Other parameters as in (Ostojic, 2014).

This is not the case in the LIF model where the "rate" and its "dynamics" depend on the temporal width over which the spiking activity is filtered. The width of the Gaussian filter used in (Ostojic, 2014) is $50 \mathrm{~ms}$. This choice is arbitrary and is the reason for the similarity observed in the rate autocorrelations (ACs) plotted in the upper and lower panels in Figure P3b which depends on this choice (Figure 1b). The rate functions were computed by filtering the spike trains of the neurons (1 ms time bin) with a Gaussian filter with $10 \mathrm{~ms}$ (the thinnest lines), $50 \mathrm{~ms}$ (moderated lines) and $100 \mathrm{~ms}$ (the thickest lines) standard deviation. Moreover, the spike ACs plotted in Figure P3c for the two models exhibit essential differences as we now show.
For $\mathrm{J}=0.2$ and $0.4 \mathrm{mV}$, the spike $\mathrm{AC}$ in the Poisson rate model (Figure P3c, upper panel) is close to a Dirac function reflecting that the dynamics are at fixed point - that is the rate variable from which the Poisson process of the spikes is generated is constant. For $\mathrm{J}=0.6 \mathrm{mV}$ the spike $\mathrm{AC}$ is very different: a broad component has now appeared. It is flat at zero time lag and has a negative curvature at short time lags (Figure 1c and Figure P3c). A detailed analysis reveals that this change has all the characteristics of a true phase transition. It shows that close to the phase transition, the amplitude vanishes proportionally to $\mathrm{J}-\mathrm{J}_{\mathrm{c}}$ and the decorrelation time diverges as $1 / \sqrt{I-J_{c}}$ (Figure S1a). To compute the ACs for the Poisson network we simulated a network for $100 \mathrm{~s}$ (time step $1 \mathrm{~ms}$ ) and 
averaged the results over 40 realizations of the initial conditions. The network size is $\mathrm{N}=100000$ for $0.5 \leq \mathrm{J} \leq 0.6 \mathrm{mV}$ and $\mathrm{N}=10000$ for $\mathrm{J}>0.6 \mathrm{mV}$. For the LIF network we averaged spike autocorrelation of 3000 randomly chosen neurons with a $1 \mathrm{~ms}$ bin following Equation 23 in the paper. All parameters are as in Figure P3.

The spike AC behaves very differently in the LIF network. For $\mathrm{J}=0.2 \mathrm{mV}$ it exhibits at zero time lag a sharp peak flanked by a trough which reflects the refractoriness (absolute and relative) of the single neuron dynamics. As $\mathrm{J}$ increases, there is a progressive change in the AC shape. Eventually, the trough disappears. The flanks of the zero peak are now decreasing exponentially (Figure 1c, solid lines). A careful analysis reveals that the typical time constant of this decrease depends only weakly on J (Figure 1c, solid lines). It is always on the order of the membrane time constant of the neurons $(20 \mathrm{~ms})$. Note also that by contrast with what is observed in the Poisson network, for $\mathrm{J}=0.5$ to $0.8 \mathrm{mV}$, the spike AC curvature is always positive and peaked around zero time lag (Figure 1c, dashed lines).

How do the "strong fluctuations" in the "heterogeneous regime" emerge? For increasing J, the spiking activity of single neurons becomes increasingly irregular, quantified by the mean coefficient of variation (cv) of the interspike interval distribution (Figure 2b). At the same time, the distribution of membrane potentials develops a very long tail towards negative voltages (Figure 2c). For strong coupling $(\mathrm{J}=0.8 \mathrm{mV})$, voltage traces of individual neurons show long very negative voltage excursions, followed by short bursts of action potentials (Figure 2d). This explains the super-Poissonian irregularity $(\mathrm{CV}>1)$. The super-Poissonian nature of spiking irregularity and the unphysiological negative voltage deviations are properties related to the linear $\dot{V}$-V-relationship of the LIF model. A mean-field description of this phenomenon requires self-consistent spike train autocorrelations (Lerchner et al., 2006; Wieland et al., 2015). For other integrate-and-fire neurons e.g. the quadraticintegrate-and-fire model, even for very strong coupling $\mathrm{J}$, e.g. $\mathrm{J}=20 \mathrm{mV}$, the mean coefficient of variation does not increase beyond one and no strongly negative voltage excursions are observed. All parameters are as in Figure P1.

Additionally, in order to compare the behavior of spiking and rate models below the postulated phase transition, we perturbed rate and spiking networks of identical topology in the least stable direction of the linearized rate dynamics, predicted by Equation 16 in the paper (Ostojic, 2014). The resulting rate deviation is projected onto the perturbation direction. The perturbation was applied to the constant feed-forward input $\mu_{0}$ for $2 \mathrm{~ms}$ where the standard deviation of the perturbation vector was $1 \mathrm{mV}$. Figure $1 \mathrm{~d}$ shows that the decay of the perturbation in the rate network slows down near the transition, indicating a critical slowing down (Figure 1d, dashed
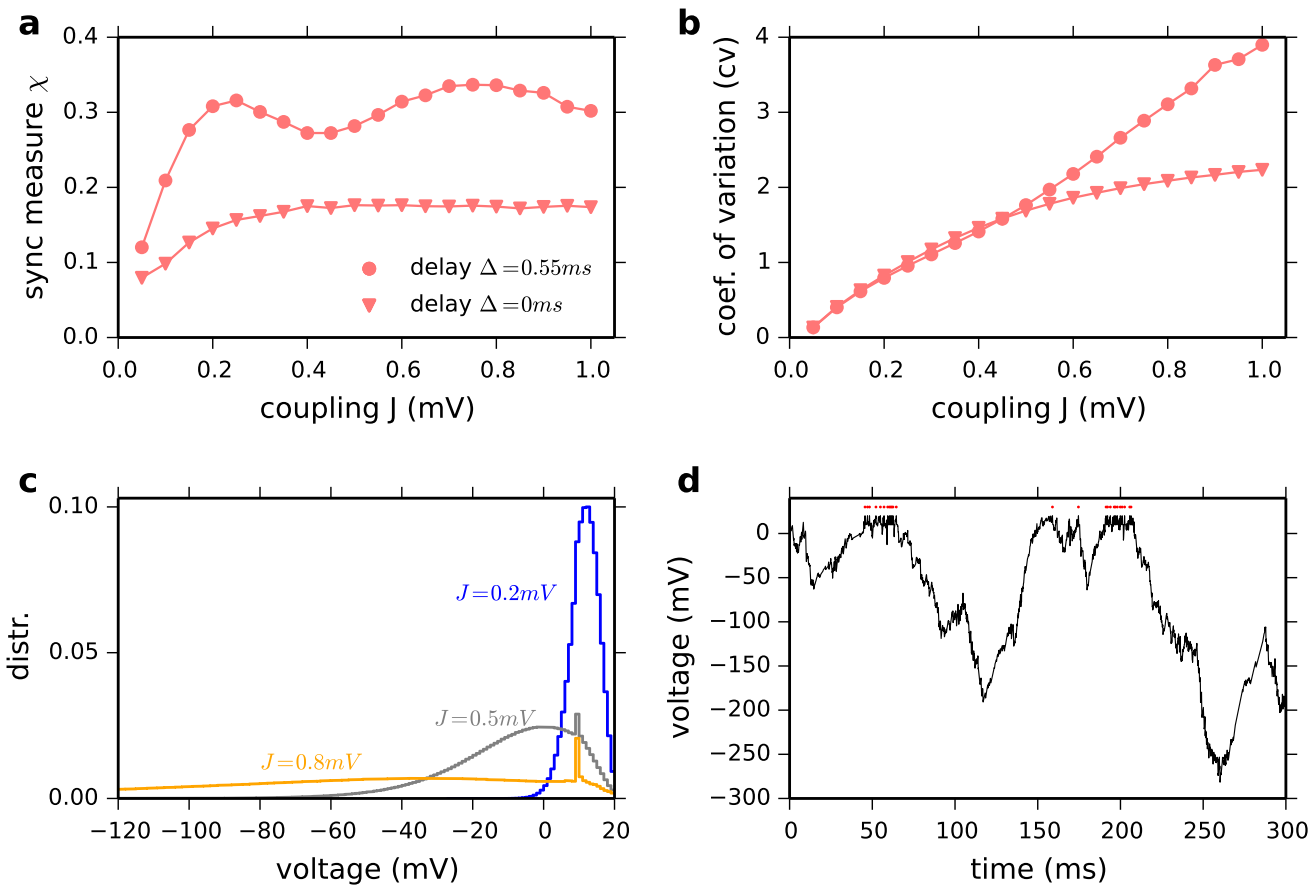

Figure 2. (a) Synchrony measure $X$ vs. coupling strength J. Dots: $\Delta=0.55 \mathrm{~ms}$. Triangles: $\Delta=0.0 \mathrm{~ms}$ for $\mathrm{N}=10000, \mathrm{C}=1000$. $\mathrm{X}$ is defined as in (Hansel \& Mato, 2003) on the phases of neurons. Note that zero delay does not increase network synchrony. (b) Coefficient of variation of the interspike intervals vs. coupling strength J. (c) Distribution of membrane potentials for different coupling strength J (in mV). (d) Example voltage trace for $\mathrm{J}=0.8 \mathrm{mV}$ shows very negative excursions followed by short bursts of action potentials. Red dots indicate spike times. Numerically exact event-based simulation were implemented in Julia programing language. Other parameters are chosen as in (Ostojic, 2014). 
lines). If there were a "mathematically analogous" transition in the spiking network, also its perturbation should decay slower as the transition is approached. Our result (Figure 1d, solid lines) shows that the decay time-scales of the perturbation (averaged over 1.42 million perturbations) is insensitive to $\mathrm{J}$ and it stays close to the membrane time constant (similar to solid lines in Figure 1c). Longer perturbation durations $(10 \mathrm{~ms})$ and weaker perturbation strengths (standard deviation $0.1 \mathrm{mV}$ ) gave very similar results (not shown). All other parameters are chosen as in (Ostojic, 2014).

\section{Conclusion}

We therefore conclude that, contrary to what was argued by the author, the spiking LIF network studied in (Ostojic, 2014) does not exhibit a phase transition to a chaotic state similar to the one occurring in the studied rate model. The reported mismatch between the average firing rate in this LIF network simulations and the mean-field calculation is unrelated to such a transition.

\section{Data and software availability}

Zenodo: Reanalysis of "Two types of asynchronous activity in networks of excitatory and inhibitory spiking neurons", 10.5281/ zenodo.59624 (Engelken \& Farkhooi, 2016).

The data for this article are also available on the Open Science Framework at: https://osf.io/q3vt4/
Author contributions

All authors participated in the research design. RE wrote the simulation and analysis code for Figure 1a,d and Figure 2a-d. FF wrote the simulation and analysis code for Figure $1 b, c$ and Figure S1a,b. Earlier simulations were performed by RE, FF, DH. All authors participated in the interpretation of the results and in manuscript writing. $\mathrm{DH}, \mathrm{CvW}$ and $\mathrm{FW}$ share joint first authorship of this article.

\section{Competing interests}

No competing interests were disclosed.

\section{Grant information}

The work of $\mathrm{DH}$ and $\mathrm{CvV}$ was partially supported by grants ANR-13-BSV4-0014-03-BALAV1 and ANR-14-NEUC-000101-BASCO and performed in the framework of the France-Israel Laboratory of Neuroscience (FILN). FF was supported by the BMBF, FKZ 01GQ 1001B. RE and FW received funding from Evangelisches Studienwerk Villigst, DFG through CRC 889 and Volkswagen Foundation.

\section{Acknowledgments}

We thank Ran Darshan, Omri Harish and Gianluigi Mongillo for fruitful discussions.

\section{Supplement material}
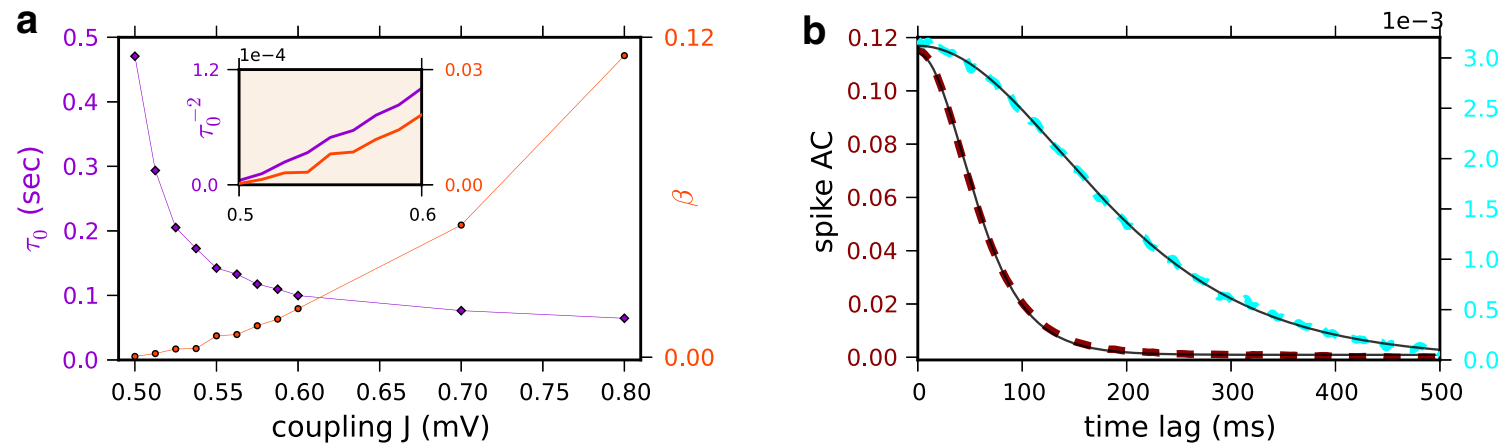

Figure S1. (a) The decorrelation time ( $\tau$, violet diamond, left y-axis) and amplitude at zero time lag (beta, orange circles, right y-axis) of the baseline-subtracted population averaged spike AC are plotted vs. $J$ for the Poisson network. These parameters were obtained by fitting the spike AC with $A C F(\tau)=\beta / \cosh \left(\tau / \tau_{0}\right)^{2}$ (see Figure S1b). Inset: the rescaled estimated $\tau_{0}^{-2}$ (left axis, violet) and $\beta$ values (orange, right axis) for $\mathrm{J}=0.5,0.5125,0.525,0.5375,0.55,0.5625,0.575,0.5875$ and $0.6 \mathrm{mV}$, to show that they vanish linearly near the phase transition. (b) The non-normalized spike $\mathrm{AC}$ can be very well fitted by $A C F(\tau)=\beta / \cosh \left(\tau / \tau_{0}\right)^{2}$. Dashed lines: Simulation results; $\mathrm{J}=0.525 \mathrm{mV}$ (cyan, right $\mathrm{y}$-axis) and $\mathrm{J}=0.8 \mathrm{mV}$ (dark red, left $\mathrm{y}$-axis): Black solid line: The fits. 
Barak O, Sussillo D, Romo R, et al:: From fixed points to chaos: three models of delayed discrimination. Prog Neurobiol. 2013; 103: 214-222.

PubMed Abstract | Publisher Full Text | Free Full Text

Churchland MM, Shenoy KV: Temporal complexity and heterogeneity of singleneuron activity in premotor and motor cortex. J Neurophysiol. 2007; 97(6): 4235-4257.

PubMed Abstract | Publisher Full Text

Engelken R, Farkhooi F: reanalysis of "Two types of asynchronous activity in networks of excitatory and inhibitory spiking neurons". Zenodo. 2016.

Data Source

Fee MS, Goldberg JH: A hypothesis for basal ganglia-dependent reinforcement learning in the songbird. Neuroscience. 2011; 198: 152-70.

PubMed Abstract | Publisher Full Text | Free Full Text

Hansel D, Mato G: Asynchronous states and the emergence of synchrony in large networks of interacting excitatory and inhibitory neurons. Neural Comput. 2003; 15(1): 1-56.

PubMed Abstract | Publisher Full Text

Harish O, Hansel D: Asynchronous Rate Chaos in Spiking Neuronal Circuits. PLoS Comput Biol. 2015; 11(7): e1004266.

PubMed Abstract | Publisher Full Text | Free Full Text

Kadmon J, Sompolinsky H: Transition to chaos in random neuronal networks.
Phys Rev X 2015: 5(4):041030.

Publisher Full Text

Lerchner A, Ursta C, Hertz J, et al:: Response variability in balanced cortical networks. Neural Comput. 2006; 18(3): 634-659.

PubMed Abstract | Publisher Full Text

Murray JD, Bernacchia A, Freedman DJ, et al:: A hierarchy of intrinsic timescales across primate cortex. Nat Neurosci. 2014; 17(12): 1661-1663.

PubMed Abstract | Publisher Full Text | Free Full Text

Ostojic S: Two types of asynchronous activity in networks of excitatory and inhibitory spiking neurons. Nat Neurosci. 2014; 17(4): 594-600.

PubMed Abstract | Publisher Full Text

Sussillo D, Abbott LF: Generating coherent patterns of activity from chaotic neural networks. Neuron. 2009; 63(4): 544-557.

PubMed Abstract | Publisher Full Text | Free Full Text

Toyoizumi T, Abbott LF: Beyond the edge of chaos: amplification and tempora integration by recurrent networks in the chaotic regime. Phys Rev E Stat Nonlin Soft Matter Phys. 2011; 84(5 Pt 1): 051908.

PubMed Abstract | Publisher Full Text

Wieland S, Bernardi D, Schwalger T, et al.: Slow fluctuations in recurrent networks of spiking neurons. Phys Rev E Stat Nonlin Soft Matter Phys. 2015; 92(4): 040901

PubMed Abstract | Publisher Full Text 


\title{
Open Peer Review
}

\section{Current Peer Review Status:}

\section{Version 1}

Reviewer Report 27 September 2016

https://doi.org/10.5256/f1000research.9839.r16565

(C) 2016 Shamir M. This is an open access peer review report distributed under the terms of the Creative Commons Attribution License, which permits unrestricted use, distribution, and reproduction in any medium, provided the original work is properly cited.

\author{
Maoz Shamir \\ Department of Physiology and Cell Biology, Ben-Gurion University of the Negev., Be'er-Sheva, \\ Israel
}

The origin and possible computational role of neuronal noise has been the focus of considerable scientific effort during the past decades. In particular, the transition to chaos has been extensively studied using simplified rate-models and much is known about this transition.

A recent work studied the dynamics of a sparsely connected network of excitatory and inhibitory spiking neurons in the balance regime and a compelling mapping between spiking neural network and rate model was proposed. Following analysis and numerical simulations it was suggested that a novel type of asynchronous state exists and it was further hypothesized that this novel state is useful for complex information processing in the central nervous system.

Here the authors reevaluate this claim. Several counter examples are provided to prove that the claim of a transition to a second type of asynchronous state does not hold. Furthermore, the origin of 'strong fluctuation' super-Poisson irregular firing is studied and is found to be related to extremely negative voltage fluctuations that are beyond the typical physiological range.

The work is timely. The results are solid and well presented. I also find the effort devoted to reproduce and re-evaluate results refreshing. I believe this paper will contribute to the scientific debate.

Competing Interests: No competing interests were disclosed.

I confirm that I have read this submission and believe that I have an appropriate level of expertise to confirm that it is of an acceptable scientific standard.

Reviewer Report 07 September 2016

https://doi.org/10.5256/f1000research.9839.r15814 
(C) 2016 Touboul J. This is an open access peer review report distributed under the terms of the Creative Commons Attribution License, which permits unrestricted use, distribution, and reproduction in any medium, provided the original work is properly cited.

\section{Jonathan Touboul}

Mathematical Neuroscience Team, CIRB - Collège de France and Inria Mycenae, Paris, France

Characterizing the dynamics of spiking neural networks and their transitions is currently a prominent issue in computational neuroscience. The question is largely non-trivial and riddled with subtleties: numerical simulations are often delicate to interpret, and theoretical tools to analyze these dynamics are still being developed.

The theoretical community have devoted important effort to address this question. Indeed, progresses on this question would advance our understanding of the brain and its computations. A question of particular interest is to characterize phase transitions to chaotic regimes in spiking networks. Indeed, since the seminal work of Sompolinsky, Crisanti and Sommers ${ }^{1}$ on rate networks, chaotic regime were shown to have rich dynamics able to support efficient computations and learning (see e.g. Sussillo \& Abbott $2009^{2}$ ). Whether spiking networks do show a similar transition and thus share similar properties as rate networks has recently been the focus of several researches and is an important endeavor in computational neuroscience ${ }^{3}$.

The present paper addresses a few important questions on the interpretations and conceptual approach of a theoretical article appeared in Nature Neuroscience in $2014^{4}$ dealing precisely with dynamics and transitions in spiking networks. That paper argued for the existence a transition in a balanced spiking network, between an asynchronous and a "new highly fluctuating regime", using in particular transitions of an associated rate model. The present article comes back to this comparison between spiking and rate network, and argues that, in contrast with the approach of Ostojic (2014) ${ }^{4}$, it is not possible to extract accurate information on the spiking network from an analysis of the particular rate network studied, by showing a mismatch between their qualitative dynamics. Moreover, using relevant numerical quantities to identify phase transitions (synchrony measure and effect of perturbations), the authors establish the absence of phase transition in the spiking network, while the rate network does present the hallmarks of phase transitions.

The present paper thus contributes to an important scientific debate on the characterization of the dynamical regimes of spiking networks. This question has been the topic of very recent important works that advance our understanding of spiking networks and the associated mean-field limits (to cite a few, see Kadmon \& Sompolinsky $2015^{5}$, Harrish \& Hansel 20156, Goedeke, Schuecker \& Helias $2016^{7}$ ).

For its contribution to the scientific debate on a timely and important topic in theoretical neuroscience, this paper shall be helpful to the readers interested in the existence and nature of transitions in spiking networks.

\section{References}

1. Sompolinsky H, Crisanti A, Sommers HJ: Chaos in random neural networks.Phys Rev Lett. 1988; 61 (3): 259-262 PubMed Abstract | Publisher Full Text

2. Sussillo D, Abbott LF: Generating coherent patterns of activity from chaotic neural networks. 
Neuron. 2009; 63 (4): 544-57 PubMed Abstract | Publisher Full Text 3. Abbott LF, DePasquale B, Memmesheimer RM: Building functional networks of spiking model neurons.Nat Neurosci. 2016; 19 (3): 350-5 PubMed Abstract | Publisher Full Text

4. Ostojic S: Two types of asynchronous activity in networks of excitatory and inhibitory spiking neurons.Nat Neurosci. 2014; 17 (4): 594-600 PubMed Abstract | Publisher Full Text

5. Kadmon J, Sompolinsky H: Transition to Chaos in Random Neuronal Networks. Physical Review $X$. 2015; 5 (4). Publisher Full Text

6. Harish O, Hansel D: Asynchronous Rate Chaos in Spiking Neuronal Circuits.PLoS Comput Biol. 2015; 11 (7): e1004266 PubMed Abstract | Publisher Full Text

7. Goedeke S, Schuecker J, Helias M: Noise dynamically suppresses chaos in random neural networks. arXiv:1603.01880 [q-bio.NC]. 2016. Reference Source

Competing Interests: No competing interests were disclosed.

I confirm that I have read this submission and believe that I have an appropriate level of expertise to confirm that it is of an acceptable scientific standard.

The benefits of publishing with F1000Research:

- Your article is published within days, with no editorial bias

- You can publish traditional articles, null/negative results, case reports, data notes and more

- The peer review process is transparent and collaborative

- Your article is indexed in PubMed after passing peer review

- Dedicated customer support at every stage

For pre-submission enquiries, contact research@f1000.com 